\title{
Investigation of a measles outbreak in Cordillera, northern Philippines, 2013
}

\author{
Paola Katrina Ching, ab Ma Justina Zapanta, ${ }^{b}$ Vikki Carr de los Reyes, ${ }^{b}$ Enrique Tayag ${ }^{b}$ and Rio Magpantay \\ Correspondence to Paola Katrina Ching (email: paolaching@gmail.com).
}

Introduction: Measles is a highly infectious viral illness that remains one of the leading causes of death among children worldwide. In the Philippines, decreasing routine vaccination coverage from 2007 to 2011 led to local measles outbreaks. A team investigated a measles outbreak reported in Cordillera of the Philippines in May 2013.

Methods: Measles case data with symptom onset from 2 February to 27 May 2013 were obtained from official sources and verified on site. Data included age, sex, residential address, signs and symptoms and vaccination status. Active casefindings were also conducted for contacts of these cases. The living environments of the cases were investigated. A survey was conducted with the cases and caregivers to understand their knowledge and attitudes about measles.

Results: There were 50 measles cases identified with an age range from six months to 32 years (median: 16 years). Thirty-two were male (64\%). Twenty (40\%) were hospitalized with one death. Thirty-two (64\%) cases were laboratory confirmed, and $36(72 \%)$ received a single dose of measles vaccine. Overcrowded living environments were observed among many cases. The majority of respondents $(46 / 48,96 \%)$ knew about measles, but there were misconceptions about the cause of measles and how it can be prevented and managed.

Conclusion: This measles outbreak occurred in an area with low immunization coverage. Achieving 95\% measles immunization coverage and strengthening routine immunization strategies to address high-risk populations are recommended. Also, we recommend health education campaigns to include components that address misconceptions about measles.

$\mathrm{M}$ easles, a highly infectious viral illness, remains one of the leading causes of death among children worldwide. ${ }^{1}$ It is caused by an RNA virus of the paramyxoviridae family which belongs to the genus morbillivirus. Its incubation period ranges from 7 to 21 days (rash appears after 14 days of exposure). ${ }^{2}$ In 2012, an estimated 122000 deaths caused by measles infections were reported globally. ${ }^{3}$

In the Philippines, measles was targeted for elimination by 2008. Routine immunization coverage of at least $95 \%$ of infants is considered the most important strategy to control measles in the country. ${ }^{4}$ The first dose of measles-containing vaccine (MCV1) is administered nine months after birth, and the second dose (MCV2) is given at 12 to 15 months. A decrease in routine measles vaccine coverage was observed in recent years. Also, the coverage of nationwide supplementary immunization activities targeting children aged nine months was $94-95 \%$ in 2007 but decreased in $2011,{ }^{5}$ probably leading to a measles outbreak in 2013.
Baguio city, a mountainous city in Benguet province of the Philippines, has a cool climate which attracts tourists the entire year. In the 2012 fully immunized child report, Baguio city had a measles immunization coverage of $78.3 \%$, while Benguet province's coverage was only $66.7 \%{ }^{6}$ On 28 May 2013 , the Philippine Event-based Surveillance and Response Unit received a report of 28 measles cases from the health officials of the Cordillera region that includes Benguet province and Baguio city. This study documents the investigation of the outbreak conducted by a team from the Philippines Field Epidemiology Training Program.

\section{METHODS}

Measles case data with symptom onset from 2 February to 27 May 2013 were obtained from a line-list prepared by the Philippine Event-based Surveillance and Response Unit. Data collected from the line-list included age, sex, residential address, signs and symptoms and vaccination status of the cases. The investigation team verified the

\footnotetext{
Field Epidemiology Training Program, Epidemiology Bureau, Department of Health, Sta Cruz, Manila, Philippines.

Department of Health, Sta Cruz, Manila, Philippines.

Submitted: 23 November 2015; Published: 11 July 2016

doi: 10.5365/wpsar.2015.6.4.007
} 
Table 1. Characteristics of the measles cases, Cordillera, Philippines, $2013(n=50)$

\begin{tabular}{|c|c|c|}
\hline Characteristics & $n$ & $\%$ \\
\hline \multicolumn{3}{|l|}{ Sex } \\
\hline Male & 32 & 64 \\
\hline Female & 18 & 36 \\
\hline \multicolumn{3}{|l|}{ Age group (years) } \\
\hline$<1$ & 3 & 6 \\
\hline $1-5$ & 9 & 18 \\
\hline $6-10$ & 3 & 6 \\
\hline $11-15$ & 19 & 38 \\
\hline $16-20$ & 11 & 22 \\
\hline $21-25$ & 1 & 2 \\
\hline$>25$ & 4 & 8 \\
\hline \multicolumn{3}{|l|}{ Case type } \\
\hline Laboratory-confirmed & 32 & 64 \\
\hline Clinically confirmed & 3 & 6 \\
\hline Epidemiologically linked & 1 & 2 \\
\hline Suspected but not confirmed & 14 & 28 \\
\hline \multicolumn{3}{|l|}{ Outcome } \\
\hline Not hospitalized & 29 & 58 \\
\hline Hospitalized & 20 & 40 \\
\hline Died & 1 & 2 \\
\hline \multicolumn{3}{|l|}{ Vaccination status } \\
\hline None & 10 & 20 \\
\hline MCV1 only & 36 & 72 \\
\hline MCV1 + MCV2 & 0 & 0 \\
\hline Unknown & 4 & 8 \\
\hline \multicolumn{3}{|c|}{ Reasons for not vaccinated $(n=10)$} \\
\hline Too young & 4 & 40 \\
\hline Belief against vaccination & 2 & 20 \\
\hline Fear of adverse events & 2 & 20 \\
\hline Forgot vaccination schedule & 1 & 10 \\
\hline Vaccine unavailability & 1 & 10 \\
\hline
\end{tabular}

$\mathrm{MCV}$, measles-containing vaccine.

measles cases and their vaccination status by visiting the listed cases on site. Active case-findings were also conducted for contacts of the listed cases.

We followed the definitions of the United States Centers for Disease Control and Prevention (CDC) for measles in this study. ${ }^{7}$ A suspected measles case was defined as a previously well individual from Cordillera with a history of fever for at least one day; generalized maculopapular rash with any of the following: cough, coryza and conjunctivitis; and with symptom onset from 2 February to 27 May 2013. A laboratory-confirmed measles case was defined as a suspected case with a positive measles immunoglobulin $\mathrm{M}$ (IgM) test. An epidemiologically linked case met the suspected case definition and was epidemiologically linked to a laboratory-confirmed case. A clinically confirmed case met the suspected case definition but with no adequate blood specimen. We obtained serum samples from the subjects and sent them to the national reference laboratory for measles IgM detection by enzymelinked immunosorbent assay (Siemens Healthineers enzygnostic anti-measles IgM test, Erlangen, Germany).

Residential environments of the cases were inspected to determine the environmental factors that might contribute to measles transmissions. Knowledge and attitudes about measles were collected through a survey by interviewing the case or his/her caregivers if the case was below 15 years.

Epi-Info version 3.5.4 (CDC, Atlanta, USA) was used for all data analyses.

\section{RESULTS}

\section{Measles cases}

There were 50 suspected measles cases identified throughout the study period. A total of 40 serum samples were collected from 40 cases; 32 of them were positive for measles lgM. The cases were not re-tested after their illness. There was also one (2\%) epidemiologically linked case and three (6\%) clinically confirmed cases. The ages of the cases ranged from six months to 32 years (median: 16 years), and there were more male $(32 / 50,64 \%)$ than female cases. The most affected age group was 11 to 15 years (19/50, 38\%). Twenty cases $(40 \%)$ were hospitalized and one died (case fatality ratio $=2 \%$ ) (Table 1 ). The majority of cases had cough $(45 / 50,90 \%)$ and coryza $(37 / 50,74 \%)$. One case developed measles one week after MCV1 vaccination. Cases started to emerge on 2 February 2013 in Benguet province and 5 March 2013 in Baguio city. The number of cases peaked during the period 4 to 10 May 2013 (Figure 1).

Thirty-six (72\%) of the 50 measles cases were vaccinated with a single dose of measles vaccine. 
Figure 1. Distribution of the measles cases by onset date of illness, Cordillera, Philippines, $2013(n=50)$

| Others* $^{*}$ Laboratory-confirmed cases

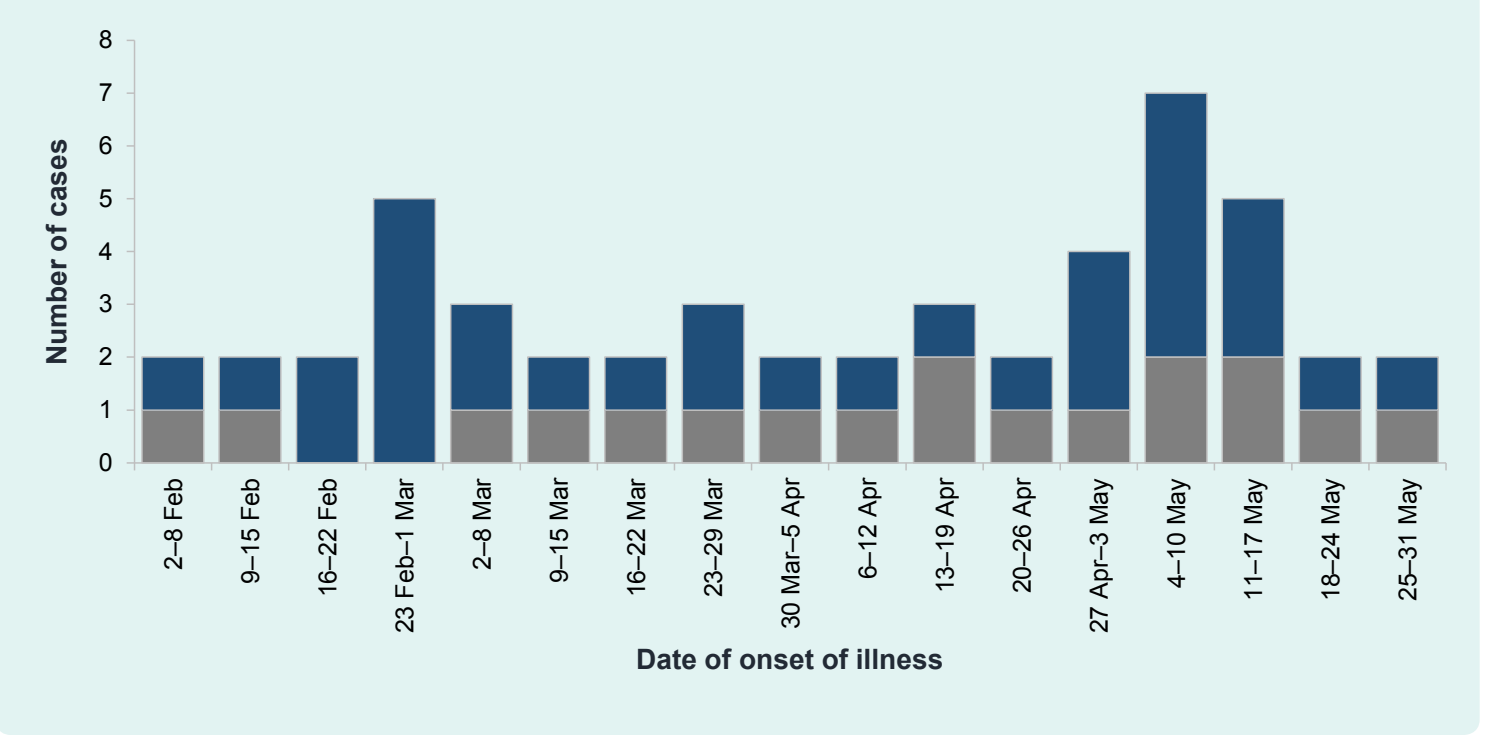

* Including suspected, clinically confirmed and epidemiologically linked cases.

Four (8\%) had an unknown vaccination history. Ten $(20 \%)$ were unvaccinated; of the unvaccinated cases, four were too young ( $40 \%)$, two (20\%) had beliefs against vaccination, two (20\%) feared adverse effects, one $(10 \%)$ forgot vaccination schedule and one (10\%) was due to vaccine unavailability (Table 1).

Sixteen measles cases (of which 12 were confirmed cases) were from one secondary school in Benguet. Twelve of them (75\%) were males aged 13 to 16 years. The index case among these 16 cases was a 14-year-old female from the same school who attended school during manifestations. Among the 16 school cases, 15 (94\%) received a single dose of measles vaccine, including one clinically confirmed case.

\section{Environmental investigation}

All the cases were living in houses that were constructed of concrete or wood or a combination of both. The houses were dim, poorly ventilated and close to each other; overcrowding was observed in most of the houses. The majority of the cases (35/50, $70 \%$ ) lived with extended families composed of four to 11 individuals. For those who were living in apartments, each floor of the apartments had one common kitchen with six to eight rooms for accommodation.

\section{Knowledge of measles}

In total, 48 subjects were interviewed. The majority $(46 / 48,96 \%)$ knew that measles is an infectious disease. Sources of information about measles were mainly relatives (20/48, 42\%), neighbours (8/48, $17 \%)$ and health centres $(8 / 48,17 \%)$. Twenty-four interviewees $(50 \%)$ did not know the cause of measles. For those who claimed that they knew the cause, their answers were unsanitary environment $(11 / 48,23 \%)$, exposure to sick person $(8 / 48,17 \%)$ and lack of sleep $(2 / 48,4 \%)$. The majority $(29 / 48,60 \%)$ believed that measles is contagious. Thirty-four (71\%) feared measles as they believed that measles is a fatal disease $(20 / 48$, $42 \%)$ and contagious (10/48, $21 \%)$. Ten (21\%) of them did not know what to do if a family member was infected with measles. Twelve $(12 / 38,32 \%)$ claimed that the family member should consult a doctor, while nine $(9 / 38,24 \%)$ said that measles patients should not be exposed to cold weather (Table 2).

\section{DISCUSSION}

Nation-wide measles resurgence in the Philippines can be attributed to the insufficient measles elimination strategies previously implemented. In this study, measles outbreaks occurred among inadequately vaccinated 
Table 2. Measles knowledge and attitudes of the measles cases and their caregivers, Cordillera, Philippines, $2013(n=48)$

\begin{tabular}{|c|c|c|}
\hline Knowledge and attitudes towards measles & $n$ & $\%$ \\
\hline \multicolumn{3}{|l|}{ Source of information about measles } \\
\hline Relatives & 20 & 42 \\
\hline Neighbours & 8 & 17 \\
\hline Health centres & 8 & 17 \\
\hline Media & 6 & 13 \\
\hline Previous measles infection & 2 & 4 \\
\hline School teacher & 2 & 4 \\
\hline Do not know & 2 & 4 \\
\hline \multicolumn{3}{|l|}{ Believe measles is contagious } \\
\hline Yes & 29 & 60 \\
\hline No & 19 & 40 \\
\hline \multicolumn{3}{|l|}{ Know the cause of measles } \\
\hline Yes - unsanitary environment & 11 & 23 \\
\hline Yes - exposure to sick person & 8 & 17 \\
\hline Yes - lack of sleep & 2 & 4 \\
\hline Yes - cold air & 2 & 4 \\
\hline Yes - poor health & 1 & 2 \\
\hline No & 24 & 50 \\
\hline \multicolumn{3}{|l|}{ Fear of measles } \\
\hline Yes - measles is a fatal disease & 20 & 42 \\
\hline Yes - measles is contagious & 10 & 21 \\
\hline Yes - others & 4 & 8 \\
\hline No & 14 & 29 \\
\hline \multicolumn{3}{|c|}{$\begin{array}{l}\text { Know what to do if a family member was infected with } \\
\text { measles }\end{array}$} \\
\hline Yes & 38 & 79 \\
\hline No & 10 & 21 \\
\hline \multicolumn{3}{|c|}{$\begin{array}{l}\text { What to do if a family member was infected with measles } \\
(n=38)^{*}\end{array}$} \\
\hline Consult a doctor & 12 & 32 \\
\hline Do not expose to cold weather & 9 & 24 \\
\hline Isolate patient & 8 & 21 \\
\hline Do not bathe & 5 & 13 \\
\hline Eat healthy foods & 3 & 8 \\
\hline Take medicine & 3 & 8 \\
\hline Eat egg & 2 & 5 \\
\hline Proper hygiene & 2 & 5 \\
\hline Wear black & 1 & 3 \\
\hline Immunization & 1 & 3 \\
\hline
\end{tabular}

* Multiple responses were allowed. groups with low measles immunization coverage. Low coverage has resulted in an accumulation of measles-susceptible children and young adults. The failure of previous campaigns to provide sufficient population immunity against measles contributed to a shift in the at-risk population from young children to various age groups. ${ }^{5}$

Insufficient measles immunization coverage in the Philippines has created the build-up of susceptible populations: those unimmunized and those who received just a single dose of measles vaccination. One dose of MCV alone cannot be attested to providing sufficient immunity. This can be further exemplified by the affected age groups: the school-age children and adolescents born before the introduction of two-dose measles vaccine in the vaccination programme in 2009. Nevertheless, a previous study showed that measles outbreaks can occur even in fully vaccinated populations. ${ }^{8}$

We revealed a cluster of measles-infected cases in a secondary school. We established exposure linkages of all student cases to the index case before their symptom onset. A previous study in Germany also reported similar age patterns for measles among the young adult age group. ${ }^{9}$ Measles infection among this age group may be associated with the absence of previous measles exposure or failure of previous measles vaccination campaigns; as in this cluster, we found nearly all cases received just a single dose of measles vaccine.

In this study, most of the measles cases were living with an extended family. Overcrowded living environments significantly increased the risk of measles infections. This confirms that increased exposure to measles cases also increases the likelihood to develop the disease. ${ }^{10}$ Also, measles viruses remain contagious in the air or on infected surfaces for up to two hours. ${ }^{11}$ The living conditions hastened the transmission through suspended droplets that are easily inhaled in closed areas.

We found that cases and caregivers had misconceptions about the cause of measles and how it 
can be prevented and managed. In India, one barrier identified for poor measles coverage was inadequate knowledge of the disease and measles vaccine. ${ }^{12}$ National programme managers should strengthen the measles education campaigns, especially to streamline those targeting hard-to-reach areas.

Several limitations had been identified in the study. Our active case-findings were limited to only contacts known by the measles cases. Also, some cases might be missed due to remote geographic locations that the investigation team could not reach. Immunization status of the subjects was only verbally validated. The majority of cases had no vaccination card or proof, and recall bias was inevitable. Despite these limitations, this outbreak investigation verifies the measles infections pattern among school-age groups and the occurrence of measles outbreaks in overcrowded areas. ${ }^{13,14}$

Based on the investigation, immunization strategies should be designed and implemented to address measles-susceptible populations in the Philippines. School-based immunization programmes for measles are recommended. Efforts should be taken to achieve 95\% MCV1 and MCV2 coverages in the country by strengthening the existing vaccination programmes. Also, we recommend health education campaigns to include components that address misconceptions about measles.

\section{Conflicts of interest}

None declared.

\section{Funding}

This outbreak investigation was funded by the Philippine Department of Health.

\section{Acknowledgements}

The author acknowledges the health staffs of Department of Health-Cordillera Administrative Region, Provincial Health Office, Municipal Health Office, City Health Office, health centres, barangay health stations, hospitals in Baguio City and Benguet, the reference laboratory of the Research Institute for Tropical Medicine, Vaccine Preventable Diseases Unit of Epidemiology Bureau and the Expanded Programme for Immunization of the
Philippine Department of Health for their collaboration and support during the investigation.

\section{References}

1. de Quadros CA. Can measles be eradicated globally? Bulletin of the World Health Organization, 2004, 82(2):134-138. pmid: 15042236

2. Fiebelkorn AP, Goodson JL. Infectious diseases related to travel. In: The Yellow Book. Atlanta, Centers for Disease Control and Prevention, 2016 (http://wwwnc.cdc.gov/travel/yellowbook/ 2016/infectious-diseases-related-to-travel/measles-rubeola, accessed 23 June 2016).

3. Measles: fact sheet. Geneva, World Health Organization, 2016 (http://www.who.int/mediacentre/factsheets/fs286/en/, accessed 23 June 2016).

4. National Objectives for Health 2005-2010. Manila, Philippines Department of Health, 2005 (http://www.doh.gov.ph/sites/default/ files/publications/NOH2005.pdf, accessed 17 June 2016).

5. Takashima $Y$ et al; Centers for Disease Control and Prevention. Progress toward measles elimination-Philippines, 1998-2014. Morbidity and Mortality Weekly Report, 2015, 64(13):357-362. pmid:25856257

6. Field Health Service Information System: Annual Report 2012. Manila, National Epidemiology Center, Department of Health, 2012 (http://www.doh.gov.ph/sites/default/files/publications/ Annual_FHSIS_2012.pdf, accessed 23 June 2016).

7. Measles. In: Hamborsky J, Kroger A, Wolfe S, eds. Epidemiology and Prevention of Vaccine Preventable Diseases, 12th Edition. Atlanta, Centers for Disease Control and Prevention, 2015, 207-228 (http://www.cdc.gov/vaccines/pubs/pinkbook/meas. html, accessed 17 June 2016).

8. Centers for Disease Control and Prevention. Measles outbreak among vaccinated high school students-Illinois. Morbidity and Mortality Weekly Report, 1984, 33(24):349-351. pmid:6427582

9. Schuster M, Stelzer T, Burckhardt F. Why are young adults affected? Estimating measles vaccination coverage in 20-34 year old Germans in order to verify progress towards measles elimination. PLoS Current, 2015, 7:7. pmid:25789202

10. Aaby $P$ et al. Determinants of measles mortality in a rural area of Guinea-Bissau: crowding, age, and malnutrition. Journal of Tropical Pediatrics. 1984 Jun;30(3):164-168. doi:10.1093/ tropej/30.3.164 pmid:6737555

11. Kutty P et al. Measles. In: Manual for the Surveillance of Vaccine Preventable Diseases. Atlanta, Centers for Disease Control and Prevention, $2014 \quad$ (http://www.cdc.gov/vaccines/pubs/survmanual/chpt07-measles.html, accessed 23 June 2016).

12. Shrivastava SR, Shrivastava PS, Ramasamy J. Measles in India: challenges and recent developments. Infection Ecology and Epidemiology, 2015, 5:27784. doi:10.3402/iee.v5.27784 pmid:26015306

13. Matson DO et al. Investigation of a measles outbreak in a fully vaccinated school population including serum studies before and after revaccination. Pediatrics Infectious Diseases Journal, 1993 12(4):292-299. doi:10.1097/00006454-199304000-00007 pmid:8483623

14. Kouadio IK, Kamigaki T, Oshitani H. Measles outbreaks in displaced populations: a review of transmission, morbidity and mortality associated factors. BMC International Health and Human Rights, 2010, 10(1):5. doi:10.1186/1472-698X-10-5 pmid:20298611 